Review began 08/17/2021 Review ended 08/31/2021 Published 09/06/202

(๑) Copyright 2021

Takakusagi et al. This is an open access article distributed under the terms of the Creative Commons Attribution License CCBY 4.0., which permits unrestricted use, distribution, and reproduction in any medium, provided the original author and source are credited.

\section{Validation of the Reliability of Machine Translation for a Medical Article From Japanese to English Using DeepL Translator}

Yosuke Takakusagi ${ }^{1}$, Takahiro Oike ${ }^{2}$, Katsuyuki Shirai ${ }^{3}$, Hiro Sato ${ }^{2}$, Kio Kano ${ }^{1}$, Satoshi Shima ${ }^{1}$, Keisuke Tsuchida ${ }^{1}$, Nobutaka Mizoguchi ${ }^{1}$, Itsuko Serizawa ${ }^{1}$, Daisaku Yoshida ${ }^{1}$, Tadashi Kamada ${ }^{1}$, Hiroyuki Katoh ${ }^{1}$

1. Department of Radiation Oncology, Kanagawa Cancer Center, Yokohama, JPN 2. Department of Radiation Oncology, Gunma University Graduate School of Medicine, Maebashi, JPN 3. Department of Radiology, Saitama Medical Center, Jichi Medical University, Omiya, JPN

Corresponding author: Yosuke Takakusagi, y-takakusagi@kcch.jp

\section{Abstract \\ Background}

The reliability of DeepL Translator (DeepL GmbH, Cologne, Germany) for the translation for medical articles has not been verified yet. In this study, we investigated the accuracy of machine translation from Japanese to English for a medical article using the DeepL Translator.

\section{Methodology}

The subject of this study was an English-language medical article translated from Japanese, which had already been published. The original Japanese manuscript was translated into English using DeepL Translator. The translated English article was then back-translated into Japanese by three researchers. In turn, three other researchers compared the back-translated Japanese sentences with the original Japanese manuscript and calculated the percentage of sentences that retained the intended meaning.

\section{Results}

The mean \pm standard deviation of the match rate for the entire article was $94.0 \pm 2.9 \%$. The match rate in the Results section was significantly higher than that in the other sections; while the match rate in the Materials and Methods section was significantly lower than the rate in the other sections. Compound sentences and sentences with an unclear subject and predicate appeared to be significant predictors for mismatched translation.

\section{Conclusions}

The translation for a medical article from Japanese to English was performed accurately by DeepL Translator.

Categories: Other

Keywords: reliability, machine translation, back translation, deepl translator, japanese to english

\section{Introduction}

Approximately 50\% of all international medical articles originate from non-English-speaking countries [1]. The share of medical articles published from Japan is only $6 \%$ and is gradually declining [1]. Writing medical articles in English is one of the obstacles faced by non-English-speaking researchers.

Machine translation (MT) is a system that automatically translates one language into another. The quality of MT has been improving, and it is widely used in various fields, such as business and translation services [2]. MT reduces the time required for translation by $95 \%$ of that required for manual translation [3]. Conventional MT uses the statistical machine translation (SMT) framework [4]. This approach employs a large amount of parallel text of the target language pair to train SMT models. Recently, MT systems employing different approaches, such as neural networks, have been developed [2]. Several studies have demonstrated that MT using neural networks shows higher translation accuracy in comparison with conventional SMT systems [5,6].

DeepL Translator (DeepL GmbH, Cologne, Germany) is one of the MT applications employing neural network systems. It was launched in August 2017, and its Japanese translation service was launched in March 2020 [7]. DeepL Translator aims to provide accurate MT. However, the reliability of DeepL Translator in translating medical articles has not yet been verified. Therefore, in this study, we investigated the 


\section{Materials And Methods}

\section{Subject}

As the subject of this study, a medical article entitled "Dosimetric Comparison Between Carbon-Ion Radiotherapy and Photon Radiotherapy for Stage I Esophageal Cancer” was used [8]. This article was published in a MEDLINE-indexed, English peer-reviewed scientific journal. It describes a dose-volume analysis that is one of the major topics of interest in the field of radiation oncology. For this article, an original draft written in Japanese (hereafter "OJ") is available.

\section{Back translation and calculation of the match rate}

Figure 1 shows an overview of the study design. The subject article (i.e., OJ) was translated from Japanese to English using the DeepL Translator in March 2021. The OJ was divided into four sections: Introduction, Materials and Methods, Results, and Discussion. MT using DeepL Translator was performed for each section. The automatically translated English text was back-translated into Japanese by translators $[9,10]$. The method of the back translation was as follows. An inappropriate word was translated as is. The translation was performed as literal translation, not free translation. If the translation was not possible, it was marked as "untranslatable." The re-translated Japanese text was termed as the back-translated manuscript in Japanese (BTJ). In turn, the judges compared the OJ and BTJ for each sentence and determined whether the meanings were consistent. The number of sentences with matched meaning was counted, and the match rate of BTJ to OJ was calculated. The match rates were calculated for the entire text and each of the four sections, namely, Introduction, Materials and Methods, Results, and Discussion.

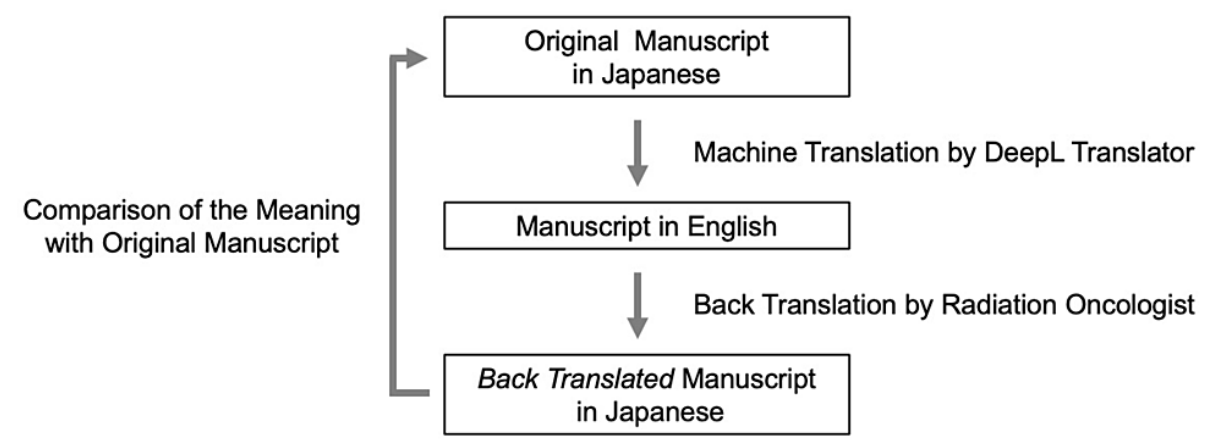

FIGURE 1: Overview of the study procedure.

\section{Translators and judges}

Three translators individually performed the back translation from English to Japanese. The eligibility criteria for the translators were as follows: (i) a native Japanese speaker; (ii) a radiation oncology expert certified by the Japanese Society for Radiation Oncology; and (iii) five or more publications in English peerreviewed scientific journals as the first author. Translators were not allowed to read the OJ in advance. Three judges individually performed their task. The judges met the following eligibility criteria: (i) a native Japanese speaker; (ii) not the translator of this study; and (iii) a radiation oncologist. The judges were blinded to the translators' information.

\section{Statistical analysis}

Differences in match rates between each section were evaluated using the Mann-Whitney U test. The association between match rates and predictors was evaluated using logistic regression analysis. A p-value of $<0.05$ was considered statistically significant. Statistical analysis was performed using Stata software version 13.1 (StataCorp LLC, College Station, TX, USA).

\section{Results}

Table 1 shows the match rates between OJ and BTJ. The total number of sentences examined was 121 . The number of sentences in the Introduction, Materials and Methods, Results, and Discussion sections was 19, 31,23 , and 48 , respectively. No sentences were determined by the judges to be untranslatable. The mean \pm standard deviation (SD) of the match rate for all the sentences was $94.0 \pm 2.9 \%$. The mean \pm SD of the match rate for the Results section was $100 \pm 0 \%$. The match rate in the Results section was significantly higher than the rates in the Introduction, Materials and Methods, and Discussion sections $(\mathrm{p}=0.004,0.001$, and 0.001 , respectively). The mean \pm SD of match rate in the Materials and Methods section was $89.2 \pm 3.9 \%$. The match rate in the Materials and Methods section was significantly lower than the rates in the Introduction, Results, 


\section{Cureus}

and Discussion sections ( $\mathrm{p}=0.028,0.001$, and 0.011 , respectively). In the Introduction and Discussion sections, the match rates were not significantly different $(\mathrm{p}=0.391)$.

\begin{tabular}{|c|c|c|}
\hline Section & Number of sentences & Match rate: mean \pm SD (\%) \\
\hline Total & 121 & $94.0 \pm 2.9$ \\
\hline Introduction & 19 & $94.7 \pm 5.3$ \\
\hline Materials and Methods & 31 & $89.2 \pm 3.9$ \\
\hline Results & 23 & $100 \pm 0$ \\
\hline Discussion & 48 & $94.2 \pm 4.6$ \\
\hline
\end{tabular}

TABLE 1: Match rate with the original manuscript in Japanese.

SD: standard deviation

Among all the sentences in the BTJ, the total number of sentences determined to be discordant by two or more judges was 12. Among these, six had unclear subject and predicate statements in the OJ. There were three compound sentences in the OJ. The number of sentences with problems in English translation was three. The number of sentences with English translation errors was three, all of which were errors in the translation of technical terms. Back translation errors were found in two sentences.

Table 2 shows the results of the statistical analysis of the predictors for translation mismatch. Although the number of letters was not a significant predictable factor for a mismatch, a compound sentence was a significant predictor of mismatch in both univariate and multivariate analyses. Odds ratios [95\% confidence interval $(\mathrm{CI})]$ in univariate and multivariate analyses were $5.23(1.52-18.02 ; \mathrm{p}=0.009)$ and $5.38(1.06-27.31$; $\mathrm{p}=0.042)$, respectively. Sentences with unclear subject and predicate were significant predictors of mismatch in both univariate and multivariate analyses. Odds ratios $(95 \% \mathrm{CI})$ in univariate and multivariate analyses were $11.11(2.96-41.64 ; \mathrm{p}<0.001)$ and $7.60(2.54-43.37 ; \mathrm{p}=0.001)$, respectively.

\begin{tabular}{|c|c|c|c|c|c|c|}
\hline & \multicolumn{3}{|c|}{ Univariate } & \multicolumn{3}{|c|}{ Multivariate } \\
\hline & OR & $(95 \% \mathrm{Cl})$ & P-value & OR & $(95 \% \mathrm{Cl})$ & P-value \\
\hline Number of letters & 1.03 & $(0.99-1.07)$ & 0.170 & 0.77 & $(0.15-4.08)$ & 0.766 \\
\hline Compound sentences & 5.23 & $(1.52-18.02)$ & 0.009 & 5.38 & $(1.06-27.31)$ & 0.042 \\
\hline Unclear subject and predicate & 11.11 & $(2.96-41.64)$ & $<0.001$ & 7.60 & $(2.54-43.37)$ & 0.001 \\
\hline
\end{tabular}

TABLE 2: Statistical analysis of predictors of translation mismatch.

OR: odds ratios; $\mathrm{Cl}$ : confidence interval

\section{Discussion}

This study investigated the accuracy of the English translation by DeepL Translator. The comparison between OJ and BTJ showed a high match rate of the meanings, suggesting high reliability of Japanese to English translation. To the best of our knowledge, this is the first study globally to investigate the accuracy of MT using DeepL Translator for medical articles.

The difference in the basic grammatical structure between two languages has been reported as a negative factor for the accuracy of conventional MT [2,3]. On the other hand, in this study, MT using DeepL Translator showed high accuracy even when the basic grammatical structure was different, such as that of Japanese and English. MT using DeepL Translator may ensure the accuracy of translation even when the basic grammatical structure of two languages differs.

Longer sentences have been reported to be a negative factor for the accuracy of MT [11]. In this study, there was no significant relationship between the length of the sentence and the accuracy of the translation; nevertheless, compound sentences and unclear subject and predicate were predictable factors for mismatch 
translations. Regarding the mistranslation of technical terms, it is imperative to use precise English words in OJ to avoid the mistranslation of technical terms. Therefore, to improve the accuracy of the translation by DeepL Translator, we recommend the following suggestions while writing OJ: clarify the subject and predicate, avoid using compound sentences as much as possible, and describe the English technical term, if necessary.

There are several limitations to this study, namely, the small sample size and subjective evaluation method. In addition, some translation errors cannot be detected by this research methodology. For example, some unsuitable words for scientific articles, such as “about" instead of "approximately," are used in the translated manuscript; and words in passive and active voices are not consistent throughout the entire manuscript. To correct these errors, proofreading the translated English manuscript is crucial.

\section{Conclusions}

In this study, DeepL Translator performed accurate MT for a medical article from Japanese to English Compound sentences and unclear subject and predicate were significant negative predictors of translation accuracy in using DeepL Translator. DeepL Translator is expected to greatly reduce the barriers in academic writing in English for non-English-speaking researchers.

\section{Additional Information \\ Disclosures}

Human subjects: All authors have confirmed that this study did not involve human participants or tissue. Animal subjects: All authors have confirmed that this study did not involve animal subjects or tissue. Conflicts of interest: In compliance with the ICMJE uniform disclosure form, all authors declare the following: Payment/services info: All authors have declared that no financial support was received from any organization for the submitted work. Financial relationships: All authors have declared that they have no financial relationships at present or within the previous three years with any organizations that might have an interest in the submitted work. Other relationships: All authors have declared that there are no other relationships or activities that could appear to have influenced the submitted work.

\section{Acknowledgements}

We would like to express our appreciation to Dr. Kouji Yamamoto, associate professor of the Department of Biostatistics, Yokohama City University School of Medicine, for his invaluable suggestions on the design of this study. The first draft of this article was prepared in Japanese and was then translated into English by DeepL Translator followed by proofreading.

\section{References}

1. Murakami A, Igami M: Benchmarking scientific research. National Institute of Science and Technology Policy, Tokyo, Japan; 2019. 10.15108/rm284http://doi.org/10.15108/rm284.

2. Dew KN, Turner AM, Choi YK, Bosold A, Kirchhoff K: Development of machine translation technology for assisting health communication: a systematic review. J Biomed Inform. 2018, 85:56-67. 10.1016/j.jbi.2018.07.018

3. Turner AM, Bergman M, Brownstein M, Cole K, Kirchhoff K: A comparison of human and machine translation of health promotion materials for public health practice: time, costs, and quality. J Public Health Manag Pract. 2014, 20:523-9. 10.1097/PHH.0b013e3182a95c87

4. Turner AM, Dew KN, Desai L, Martin N, Kirchhoff K: Machine translation of public health materials from English to Chinese: a feasibility study. JMIR Public Health Surveill. 2015, 1:e17. 10.2196/publichealth.4779

5. Wu C, Xia F, Deleger L, Solti I: Statistical machine translation for biomedical text: are we there yet? . AMIA Annu Symp Proc. 2011, 2011:1290-9.

6. Wu Y, Schuster M, Chen $\mathrm{Z}$ et al.: Google's neural machine translation system: bridging the gap between human and machine translation. ArXiv. 2016, 1609:08144

7. DeepL Translator. (2020). Accessed: March 19, 2020: https://www.deepl.com/en/blog/20200319.html.

8. Takakusagi Y, Yoshida D, Kusano Y, et al.: Dosimetric comparison between carbon-ion radiotherapy and photon radiotherapy for stage I esophageal cancer. In Vivo. 2021, 35:447-52. 10.21873/invivo.12277

9. Valko L, Baglyas S, Kunos L, Terray-Horvath A, Lorx A, Gal J, Windisch W: Validation of the Hungarian version of the SRI Questionnaire. BMC Pulm Med. 2020, 20:130. 10.1186/s12890-020-1171-5

10. Dasrilsyah RA, Ng BK, Atan IK, Khong SY, Nusee Z, Lim PS: Validation of the Malay version of the p-QOL questionnaire [In Press]. Int Urogynecol J. 2020, 10.1007/s00192-020-04362-W

11. Kiuchi T, Kaihara S: Quantitative evaluation of English-Japanese machine translation of medical literature Methods Inf Med. 1991, 30:199-205. 